\title{
Black polarization sandwiches are square roots of zero
}

\author{
M V Berry and M R Dennis
}

H H Wills Physics Laboratory, University of Bristol, Tyndall Avenue, Bristol BS8 1TL, UK

Received 29 July 2003, accepted for publication 28 August 2003

Published 24 February 2004

Online at stacks.iop.org/JOptA/6/S24 (DOI: 10.1088/1464-4258/6/3/004)

\begin{abstract}
In the $2 \times 2$ matrices representing retarders and ideal polarizers, the eigenvectors are orthogonal. An example of the opposite case, where eigenvectors collapse onto one, is matrices $\mathbf{M}$ representing crystal plates sandwiched between a crossed polarizer and analyser. For these familiar combinations, $\mathbf{M}^{2}=0$, so black sandwiches can be regarded as square roots of zero. Black sandwiches illustrate physics associated with degeneracies of non-Hermitian matrices.
\end{abstract}

Keywords: polarization, degeneracy, nilpotence, matrix optics

Tudor [1, 2] has recently remarked that the common classification of polarizing devices into retarders, represented by unitary operators, and ideal polarizers, represented by projection operators, fails to include some commonly used combinations of optical elements. Both retarders and ideal polarizers are represented by $2 \times 2$ Jones matrices [3-5] whose eigenvectors are orthogonal; the difference lies in the eigenvalues: for retarders, two complex eigenvalues lie on the unit circle, and for ideal polarizers, one eigenvalue is zero. Tudor gives the example of a linear polarizer followed by a quarter-wave plate, where the eigenvectors are not orthogonal. He also gives an example of the extreme case of nonorthogonal eigenvectors, namely parallel eigenvectors: a linear polarizer sandwiched between two identical quarterwave plates oriented at $45^{\circ}$ to the axes of the polarizer; this example falls into a class described by de Lang [6].

Here we extend Tudor's remark in a very simple way, by pointing out that this extreme case of parallel eigenvectors is realized optically by a much wider class of devices including some of the most familiar combinations employed in polarization optics, namely any specimen (e.g. a crystal plate) between a crossed polarizer and analyser-which we call a black sandwich, for obvious reasons. The matrix for a black sandwich is

$$
\mathbf{M}=\mathbf{P}_{+} \mathbf{A} \mathbf{P}_{-},
$$

where

$$
\mathbf{P}_{ \pm}=| \pm\rangle\langle \pm|
$$

are the projection matrices corresponding to the ideal polarizer $(-)$ and analyser $(+)$ that select orthogonal states represented by the column (e.g. Jones) vectors $| \pm\rangle$, and $\mathbf{A}$, representing the specimen, can be any $2 \times 2$ matrix.
Because of its unsymmetrical form (polarizer and analyser different), $\mathbf{M}$ is non-Hermitian, and explicit calculation shows that both of its eigenvalues are zero. Therefore the two eigenvectors, that are different for the general case, have here collapsed onto one, namely $|+\rangle$. A polarization $|\psi\rangle$ entering the black sandwich emerges in the state

$$
\mathbf{M}|\psi\rangle=(\langle+|\mathbf{A}|-\rangle\langle-\mid \psi\rangle)|+\rangle .
$$

In contrast to the ideal polarizer $\mathbf{P}_{+}$, from which the emerging light is also in the state $|+\rangle$ but which extinguishes incident light in the orthogonal state $|-\rangle$, the black sandwich extinguishes its own eigenstate. This is obvious when $\mathbf{M}$ is written in the form

$$
M=(\langle+|A|-\rangle)|+\rangle\langle-| \text {. }
$$

It follows immediately that

$$
\mathbf{M}^{2}=0,
$$

i.e. $\mathbf{M}$ is nilpotent, reflecting the obvious fact that the combination of two black sandwiches extinguishes all light. Therefore black sandwiches (1), incorporating general matrices $\mathbf{A}$, can be regarded as nontrivial square roots of zero. (The trivial square root $\mathbf{M}=0$ corresponds to $\mathbf{A}=1$ - simply a crossed polarizer and analyser, i.e. a sandwich with no filling.)

A familiar black sandwich consists of a transparent crystal plate between a linear polarizer and analyser. If the polarizer and analyser are

$$
\begin{aligned}
& |-\rangle=\left(\begin{array}{l}
0 \\
1
\end{array}\right) \quad \text { (polarizer) } \\
& |+\rangle=\left(\begin{array}{l}
1 \\
0
\end{array}\right) \quad \text { (analyser) }
\end{aligned}
$$


and the orthogonal eigenpolarizations and eigenvalues of the crystal are

$$
\begin{gathered}
\left|\phi_{1}\right\rangle=\left(\begin{array}{c}
u \\
v
\end{array}\right), \quad \text { eigenvalue } \lambda_{0}+\lambda, \\
\left|\phi_{2}\right\rangle=\left(\begin{array}{c}
v^{*} \\
-u^{*}
\end{array}\right), \quad \text { eigenvalue } \lambda_{0}-\lambda,
\end{gathered}
$$

with $|u|^{2}+|v|^{2}=1$, then $\mathbf{A}$ is the unitary matrix

$$
\begin{aligned}
\mathbf{A} & =\exp \left(\mathrm{i} \lambda_{0}\right)\left[\left|\phi_{1}\right\rangle\left\langle\phi_{1}|\exp (\mathrm{i} \lambda)+| \phi_{2}\right\rangle\left\langle\phi_{2}\right| \exp (-\mathrm{i} \lambda)\right] \\
& =\exp \left(\mathrm{i} \lambda_{0}\right)\left[\cos \lambda \mathbf{I}+\mathrm{i} \sin \lambda\left(\begin{array}{cc}
|u|^{2}-|v|^{2} & 2 u v^{*} \\
2 u^{*} v & |v|^{2}-|u|^{2}
\end{array}\right)\right],
\end{aligned}
$$

and the black sandwich matrix is

$$
\mathbf{M}=\langle+|\mathbf{A}|-\rangle|+\rangle\langle-|=2 \mathrm{i} u v^{*} \exp \left(\mathrm{i} \lambda_{0}\right) \sin \lambda\left(\begin{array}{ll}
0 & 1 \\
0 & 0
\end{array}\right)
$$

For an anisotropic material, the polarization components $u$ and $v$ depend on direction, and any diffuse light (e.g. the sky, or white paper) viewed through the sandwich will exhibit the familiar conoscopic figures [7, 8]. An easy way to see the conoscopic figures displaying the polarization singularity at the optic axis of a biaxial material is with a 'crystal' consisting of a sheet of overhead-projector transparency film [9], so this 'black plastic sandwich' is a square root of zero. For conoscopic figures corresponding to more general polarization singularities, with $\mathbf{A}$ representing crystals that are gyrotropic and dichroic as well as birefringent, see [10].

The de Lang class [6] of nilpotent devices, mentioned at the end of the first paragraph, is more restricted, being of the form $\mathbf{M}=\mathbf{U P U}$, where $\mathbf{U}$ is a quarter-wave retarder whose eigenpolarizations are directions on the Poincaré sphere perpendicular to that selected by $\mathbf{P}$. This class forms a four-parameter family of devices (including overall phase), whereas the black sandwiches form a ten-parameter family (or, if $\mathbf{A}$ is restricted to the class of retarders, a six-parameter family).

The polarization optics of black sandwiches joins a growing class of physics associated with the collapse of two eigenvectors onto one at a degeneracy of eigenvalues of non-Hermitian matrices. Other examples occur in the diffraction of atoms by 'crystals of light' [11-13], in nuclear physics [14-17], and in the linewidths of unstable lasers [18]. Such degeneracies also occur in the optics of absorbing crystals [8, 10, 19-21], for light travelling along a 'singular axis' (to avoid confusion, we emphasize that in (1) these degeneracies occur in the crystal matrix $\mathbf{A}$, not the black sandwich matrix $\mathbf{M}$ ).

\section{Acknowledgments}

MVB is supported by the Royal Society, and MRD is supported by the Leverhulme Trust.

\section{References}

[1] Tudor T 2003 Generalized observables in polarization optics J. Phys. A: Math. Gen. 36 9577-90

[2] Tudor T 2003 ICO Topical Meeting on Polarization Optics (Physics Department Selected Papers 8) ed A A Friesem and J Turunen (Polvijärvi: University of Joensuu) pp 46-47

[3] Azzam R M A and Bashara N M 1977 Ellipsometry and Polarized Light (Amsterdam: North-Holland)

[4] Berry M V and Klein S 1996 Geometric phases from stacks of crystal plates J. Mod. Opt. 43 165-80

[5] Brosseau C 1998 Fundamentals of Polarised Light: a Statistical Optics Approach (New York: Wiley)

[6] de Lang H 1967 Polarization properties of optical resonators passive and active Philips Res. Rep. 37 Suppl. 1-67

[7] Born M and Wolf E 1959 Principles of Optics (London: Pergamon)

[8] Ramachandran G N and Ramaseshan S 1961 Crystal optics Handbuch der Physik vol XXV/I, ed H Flügge (Berlin: Springer)

[9] Berry M V, Bhandari R and Klein S 1999 Black plastic sandwiches demonstrating biaxial optical anisotropy Eur. J. Phys. 20 1-14

[10] Berry M V and Dennis M R 2003 The optical singularities of birefringent dichroic chiral crystals Proc. R. Soc. A 459 1261-92

[11] Oberthaler M K, Abfalterer R, Bernet S, Schmiedmayer J and Zeilinger A 1996 Atom waves in crystals of light Phys. Rev. Lett. 77 4980-3

[12] Berry M V and O’Dell D H J 1998 Diffraction by volume gratings with imaginary potentials J. Phys. A: Math. Gen. 31 2093-101

[13] Berry M V 1998 Lop-sided diffraction by absorbing crystals J. Phys. A: Math. Gen. 31 3493-502

[14] Heiss W D 2000 Repulsion of resonance states and exceptional points Phys. Rev. E 61 929-32

[15] Heiss W D and Harney H L 2001 The chirality of exceptional points Eur. Phys. J. D 17 149-51

[16] Rotter I 2001 Correlations in quantum systems and branch points in the complex plane Phys. Rev. C 64034301

[17] Rotter I 2002 Branch points in the complex plane and geometric phases Phys. Rev. E 65026217

[18] Berry M V 2003 Mode degeneracies and the Petermann excess-noise factor for unstable lasers J. Mod. Opt. 50 63-81

[19] Pancharatnam S 1955 The propagation of light in absorbing biaxial crystals-I. Theoretical Proc. Indian Acad. Sci. 42 86-109

[20] Pancharatnam S 1955 The propagation of light in absorbing biaxial crystals-II. Experimental Proc. Indian Acad. Sci. 42 235-48

[21] Berry M V 1994 Pancharatnam, virtuoso of the Poincaré sphere: an appreciation Curr. Sci. 67 220-23 